Homology, Homotopy and Applications, vol.15(1), 2013, pp.313-342

\title{
POWER OPERATIONS IN ORBIFOLD TATE $K$-THEORY
}

\author{
NORA GANTER
}

\author{
(communicated by J. Daniel Christensen)
}

\begin{abstract}
We formulate the axioms of an orbifold theory with power operations. We define orbifold Tate $K$-theory, by adjusting Devoto's definition of the equivariant theory, and proceed to construct its power operations. We calculate the resulting symmetric powers, exterior powers and Hecke operators and put our work into context with orbifold loop spaces, level structures on the Tate curve and generalized Moonshine.
\end{abstract}

\section{Introduction}

Tate $K$-theory,

$$
K_{\text {Tate }}(X)=K(X)[[q]]
$$

is a form of elliptic cohomology. The corresponding generalized elliptic curve is the Tate curve, and the corresponding elliptic genus is the Witten genus [4]. The most conceptual definition of the Tate curve is probably the characterization in $[\mathbf{1 3}$, Thm. VII.2.1]: if $\mathcal{M}_{\text {ell }}$ is the algebraic stack of (nice enough) generalized elliptic curves, ${ }^{1}$ then the completion of $\mathcal{M}_{\text {ell }}$ at infinity,

$$
\operatorname{spf} \mathbb{Z} \llbracket q \rrbracket \longrightarrow \mathcal{M}_{\mathrm{ell}},
$$

classifies a formal projective curve. This possesses an algebraization, living over $\operatorname{spec} \mathbb{Z} \llbracket q \rrbracket$, which we will call $\operatorname{Tate}(q)$. A more pedestrian, but equivalent, formulation of this definition of $\operatorname{Tate}(q)$ can be found in [4].

Out of all known elliptic cohomology theories, $K_{\text {Tate }}$ might be the one where the conjectural relationship to string theory is best understood, and Witten's original definition of his genus involves loop spaces.

The definition of $G$-equivariant Tate $K$-theory for finite $G$ goes back to Devoto [14] and is modeled on the loop space of a global quotient orbifold. Our definition of

The author is supported by a Discovery Grant from the Australian Research Council and currently holds an Australian Research Fellowship. This paper extends work that the author pursued in 2006, during a stay at MSRI and the subsequent time at University of Illinois. At that time, she was supported by NSF grant DMS-0504539.

Received July 27, 2009, revised November 12, 2012; published on May 31, 2013.

2000 Mathematics Subject Classification: 19L99.

Key words and phrases: Elliptic cohomology, Tate curve, cohomology operation, level structure, generalized moonshine.

Article available at http://intlpress . com/HHA/v15/n1/a16 and doi:10.4310/HHA .2013.v15.n1.a16

Copyright (C) 2013, International Press. Permission to copy for private use granted.

${ }^{1}$ We write $\mathcal{M}_{\text {ell }}$ for the stack denoted $\mathcal{M}_{1}$ in [13, III.2.6]. 
orbifold Tate $K$-theory is a mild modification of Devoto's. Devoto's work also already makes the connection to level structures on elliptic curves.

Guided by the ideas in $\left[\mathbf{1 5}\right.$, we define power operations on $K_{\text {Tate }}$. This project grew out of the desire for a more explicit link between the findings in [16] and the string theoretic picture in $[\mathbf{1 5}]$ and was inspired by the similarities between $[\mathbf{1 5}]$ and $[\mathbf{2 4}, \mathbf{3 4}]$.

The story in $[\mathbf{1 6}]$ is one of convergence: there, we saw how the purely homotopy theoretic formalism of power operations in elliptic cohomology (the theory there is $\left.E_{2}\right)$ results in a formula much resembling that of Dijkgraaf, Moore, Verlinde and Verlinde.

Here, we take a more traditional approach to mathematical physics, modeling the mathematical definitions on the calculations of physicists. The resulting power operations are closely linked to level structures and isogenies on the Tate curve, suggesting that this is the 'right' definition of elliptic power operations on $K_{\text {Tate }}$. To make precise exactly what is meant by 'right' in this context, one has to revisit the work of Matthew Ando, his power operations on $K_{\text {Tate }}$, and the definition of elliptic power operations in [5]. All this was pioneered in the work of Andrew Baker, who was the first to define Hecke operators on elliptic cohomology [8, 7] and [9].

The formalism of power operations leads to a number of internal operations, acting on the Tate $K$-theory of any space. In particular, it yields symmetric powers

$$
\operatorname{Sym}_{\text {Tate }}^{n}: K_{\text {Tate }}(X) \longrightarrow K_{\text {Tate }}(X) \text {. }
$$

We will write

$$
S_{t}^{\text {Tate }}(x):=\sum_{n=o}^{\infty} \operatorname{Sym}_{\text {Tate }}^{n}(x) t^{n}
$$

for the total symmetric power. Here $t$ is a formal variable. On the coefficients of $K_{\text {Tate }}$, the total symmetric power satisfies the identity

$$
S_{t}^{\text {Tate }}(x)=\exp \left(\sum_{m \geqslant 1} T_{m}(x) t^{m}\right),
$$

where the $T_{m}$ are the equivariant Hecke operators of generalized Moonshine defined in $[\mathbf{1 7}, \mathbf{2 9}]$ and prominent in Carnahan's proof of the generalized Moonshine Conjectures [12]. The identity (1) gives rise to a new formulation of the replicability condition, although the latter remains mysterious. On the other hand, the total symmetric power of a vector bundle is the stable exponential characteristic class

$$
S_{t}^{\text {Tate }}(V)=\bigotimes_{k \geqslant 1} S_{t^{k}}(V)
$$

used to define the Witten genus. Here $S_{t}$ is the total symmetric power in $K$-theory. The Witten genus is, of course, an important player in Moonshine, because of the Hirzebruch conjecture $[\mathbf{2 1}, \mathbf{2 5}]$.

In the context of replicability, the variable $t$ in (1) lives a peculiar and poorly understood double life as a formal variable on one hand and as the modular variable $e^{2 \pi i \tau}$ on the other. In the context of (2), the same double life occurs, and a compelling explanation for this phenomenon, involving the boundary of a Krichever 
style $^{2}$ construction of the relevant moduli space, goes back to unpublished work of Looijenga [10].

\section{Acknowledgements}

It is a pleasure to thank Nitu Kitchloo, Matthew Ando, Charles Rezk, Alex Ghitza, David Gepner, Craig Westerland, Christian Haesemeyer, Ralph Cohen, Ernesto Lupercio, Bernardo Uribe, Jorge Devoto and John McKay for many helpful conversations on the topic. I would also like to thank the referee for very useful suggestions on an earlier account of the material and, in particular, for pointing out the connection to level structures.

\section{2. $\quad$ Orbifold Tate $K$-theory}

We will work in the 2-category Gpd whose objects are the (small) topological groupoids and with

$$
1 \operatorname{Hom}(X, Y)=\operatorname{Fun}(X, Y)
$$

the groupoid of continuous functors from $X$ to $Y$ and continuous natural isomorphisms between them. We do not emphasize the 2-category point of view. For all practical purposes, we could as well work in the category

$$
\operatorname{Gpd}=\operatorname{Gpd} / \cong,
$$

obtained from Gpd by identifying naturally isomorphic 1-morphisms, or even in Moerdijk's orbifold category Orb (see $[\mathbf{2 8}]$ ). The center of a groupoid $X$ is the group

$$
\operatorname{Center}(X):=2 \operatorname{Hom}\left(\operatorname{Id}_{X}, \operatorname{Id}_{X}\right)=\operatorname{Nat}\left(\operatorname{Id}_{X}, \operatorname{Id}_{X}\right),
$$

of natural transformations from $\operatorname{Id}_{x}$ to $\operatorname{Id}_{x}$. We will also need the 2-category $\operatorname{Gpd}^{\text {cen }}$ whose objects are pairs $(X, \xi)$ with $\xi$ a center element of $X$ and with

$$
1 \operatorname{Hom}((X, \xi),(Y, v)) \subset \operatorname{Fun}(X, Y)
$$

the full subcategory of functors $f$ satisfying

$$
f \xi=v f .
$$

Lemma 2.1. If $\eta: f \cong f^{\prime}$ is a natural isomorphism and $f \xi=v f$, then $f^{\prime} \xi=v f^{\prime}$.

Proof. For any $x \in \mathrm{ob}(X)$, we have

$$
v_{f^{\prime}(x)}=\eta_{f(x)} \circ v_{f(x)} \circ \eta_{f(x)}^{-1}=\eta_{f(x)} \circ f\left(\xi_{x}\right) \circ \eta_{f(x)}^{-1}=f^{\prime}\left(\xi_{x}\right) .
$$

For simplicity of exposition, all center elements are assumed to be of finite order. This is automatically the case if $X$ is an orbifold groupoid in the sense of Moerdijk. If $G$ is a finite group and $M$ is a $G$-space, then we write $M / / G$ for the corresponding translation groupoid.

${ }^{2}$ Cutting along the circle $[0,1]$ inside $\mathbb{C} /\langle\tau, 1\rangle$, instead of Krichever's small circle as in $[\mathbf{3 1}, 8.11]$. 
Example 2.2. The group

$$
\text { Center }(\mathrm{pt} / / G)=\operatorname{Center}(G)
$$

is the center of the group $G$.

Example 2.3. The inertia groupoid

$$
\Lambda X=\operatorname{Fun}(\mathrm{pt} / / \mathbb{Z}, X)
$$

of $X$ admits a group homomorphism

$$
\begin{aligned}
& \mathbb{Z} \longrightarrow \operatorname{Center}(\Lambda X) \\
& k \longmapsto\left(\xi^{k}: f \mapsto f(k)\right) .
\end{aligned}
$$

Viewing objects of $\Lambda X$ as pairs $(x, g)$ with $x \in \mathrm{ob}(X)$ and $g \in \operatorname{aut}(x)$, we have

$$
\xi_{(x, g)}^{k}=g^{k}
$$

(arrow in $\Lambda X)$.

For any $k \in \mathbb{Z}$, we then have the 2 -functor

$$
\begin{aligned}
\mathrm{Gpd} & \longrightarrow \mathrm{Gpd}^{\text {cen }} \\
X & \longmapsto\left(\Lambda X, \xi^{k}\right) .
\end{aligned}
$$

Example 2.4. In the global quotient case, we have

$$
\Lambda(M / / G) \simeq \coprod_{[g]} M^{g} / / C_{g} \quad \text { and }\left.\quad \xi^{k}\right|_{M^{g}}=g^{k} .
$$

Here $[g]$ is the conjugacy class, $M^{g}$ is the fixed point locus, and $C_{g}$ is the centralizer of $g$ in $G$.

Definition 2.5. The $k$ th power map

$$
\Pi^{k}:\left(\Lambda X, \xi^{k}\right) \longrightarrow\left(\Lambda X, \xi^{1}\right)
$$

sends the object $(x, g)$ to $\left(x, g^{k}\right)$ and the arrow $(x, g) \stackrel{h}{\longrightarrow}\left(h x, h g h^{-1}\right)$ to

$$
\left(x, g^{k}\right) \stackrel{h}{\longrightarrow}\left(h x, h g^{k} h^{-1}\right) .
$$

We may also interpret $\Pi^{k}$ as a 1 -morphism from $\left(\Lambda X, \xi^{k m}\right)$ to $\left(\Lambda X, \xi^{m}\right)$.

Definition 2.6. Let $X$ be a topological groupoid, let $\xi$ be an element of its center, and let $k$ be an integer. Then we define the groupoid

$$
X\left[\xi^{\frac{1}{k}}\right]:=(\mathrm{pt} / / \mathbb{Z}) \times \Lambda(X) / \sim,
$$

where the equivalence relation $\sim$ is generated by $k \sim \xi$.

Definition 2.7. For an object $(X, \xi)$ of $\mathrm{Gpd}^{\text {cen }}$, the subring

$$
K_{\text {rot }}(X, \xi) \subset K_{\text {orb }}(X) \llbracket q^{\frac{1}{|\xi|}} \rrbracket
$$

is the Grothendieck group of formal power series

$$
F(q)=\sum_{a \in \mathbb{Q} \geqslant 0} V_{a} q^{q}
$$


satisfying the rotation condition with respect to $\xi$ :

for each $a \in \mathbb{Q}_{\geqslant 0}$ the coefficient $V_{a}$ is an $e^{2 \pi i a}$-eigenbundle of $\xi$.

Here we are using Moerdijk's definition of orbifold $K$-theory $K_{\text {orb }}(X)$; see $[\mathbf{2 8}, 5.4]$.

Power series satisfying the rotation condition may be thought of as (infinite dimensional) vector bundles over

$$
\mathbb{R} \ltimes_{\xi} X:=(\mathrm{pt} / / \mathbb{R}) \times X / \sim
$$

with $\sim$ generated by $1 \sim \xi$.

Definition 2.8. Let $X$ be a topological groupoid. Then the Tate $K$-theory of $X$ is defined as

$$
K_{\text {Tate }}(X):=K_{\text {rot }}\left(\Lambda(X), \xi^{1}\right) .
$$

\subsection{Motivation}

2.1.1. The Tate curve

Let $S=\operatorname{spec}(\mathbb{Z} \llbracket q \rrbracket)$. Then there is an isomorphism of formal schemes over $S$

$$
\operatorname{spf}\left(K_{\text {Tate }}\left(\mathbb{C} P^{\infty}\right)\right)=\operatorname{Tate}(q) \widehat{S},
$$

making $K_{\text {Tate }}$ an elliptic cohomology theory; cf. $[4,2.6]$. Let $C_{k}:=\mathbb{Z} / k \mathbb{Z}$ be the cyclic group with $k$ elements. Then the complex representation ring of $C_{k}$ is

$$
R\left(C_{k}\right) \cong \mathbb{Z}\left[\zeta_{k}\right],
$$

where the $k$ th root $\zeta_{k}$ of one is identified with the representation where 1 acts as $e^{2 \pi i / k}$. So,

$$
\operatorname{spec} R\left(C_{k}\right) \cong \mu_{k}
$$

is the scheme of $k$ th roots of unity over $\mathbb{Z}$, where $\zeta_{k}$ is the irreducible representation with $\zeta_{k}(1)=e^{2 \pi i / k}$, and one expects ${ }^{3}$

$$
\operatorname{spec}\left(K_{\text {Tate }}\left(\operatorname{pt} / / C_{k}\right)\right)=\operatorname{Tate}(q)[k],
$$

where the right-hand side is the scheme of points of order $k$ in $\operatorname{Tate}(q)$. By $[\mathbf{1 3}$, VII(1.13)],

$$
\operatorname{Tate}(q)[k]=\operatorname{spec}\left(D_{k}\right)
$$

where

$$
D_{k}:=\bigoplus_{j=0}^{k-1} \mathbb{Z} \llbracket q \rrbracket\left[x, x^{-1}\right] / x^{k}-q^{j} .
$$

After inverting $q$, we have the map

$$
\begin{aligned}
J: \mathbb{Z}((q))[x] & \longrightarrow \mathbb{Z}\left[\zeta_{k}\right]\left(\left(q^{\frac{1}{k}}\right)\right) \\
q & \longmapsto q \\
x & \longmapsto \zeta_{k} q^{\frac{j}{k}} .
\end{aligned}
$$

${ }^{3}$ See $[3,14,17,19,20]$. 
The map $J$ identifies the $j$ th summand of $D_{k}$ with the Laurent series satisfying the rotation condition for the group element $j$. So, (4) holds over the locus $\operatorname{spec} \mathbb{Z}((q))$, where the Tate curve is non-singular. The ring $D_{k}$ is closely related to isogenies into Tate $(q)$ and plays a key role in Ando's definition of power operations on $K_{\text {Tate }}$; see $[\mathbf{2}$, p. $26 \mathrm{ff}]$.

We would also like to draw the reader's attention to Rezk's [32], where a much deeper connection between the constructions in $[\mathbf{1 3}]$ and our Definition 2.8 is discussed.

\subsubsection{Constant loops}

The original motivation for Definition 2.8 came from the theory of orbifold loop spaces. In [23], Lupercio and Uribe identify the inertia groupoid as the full subcategory

$$
\Lambda X \subseteq \operatorname{Fun}(\mathbb{R} / / \mathbb{Z}, X)
$$

of functors that are constant on objects. Viewing the right-hand side as part of the loop groupoid

$$
L X=\operatorname{Orb}\left(\mathbb{S}^{1}, X\right),
$$

then leads them to the identification

$$
\Lambda X=(L X)^{\mathbb{R}}
$$

of the inertia groupoid with the orbifold loops that are fixed by the rotation action. Under this identification, $\xi^{k}$ agrees with the rotation action of $k \in \mathbb{Z}$ on (constant) orbifold loops.

Example 2.9 (Global quotients). By [23, 4.1],

$$
L(M / / G) \simeq \coprod_{[g]} \mathcal{L}_{g} M / / C_{g}
$$

is the union of the "twisted loop spaces" of $M$, i.e., of paths in $M$ from $x$ to $g x$, acted upon by the centralizers $C_{g}$. This is what motivated Devoto's definition of equivariant Tate $K$-theory [14, pp. 5f].

In this context, the rotation condition is motivated by the following lemma. Let $V$ be a finite dimensional, real orbifold vector bundle on $X$, the $V$ may itself be viewed as a groupoid (see, e.g., [28]), and we can form $L V$.

Lemma 2.10. In this situation, we have a dense isomorphism

$$
\left.L V\right|_{\Lambda(X)} \cong V_{0} \oplus \bigoplus_{a \in \mathbb{Q}_{+}} V_{a}^{\mathbb{C}} q^{a} .
$$

Here $V_{0}$ is the summand of $\left.V\right|_{\Lambda(X)}$ fixed by $\xi^{1}$, and for $a \in \mathbb{Q}_{+}$, the bundle $V_{a}^{\mathbb{C}}$ is the $e^{2 \pi i a}$-eigenbundle of $\xi^{1}$ inside the complexification $V_{\Lambda(X)}^{\mathbb{C}}$, while $q^{a}$ indicates that $\mathbb{R}$ acts on this summand via $t \mapsto e^{2 \pi i a t}$. 
Proof. Let $(x, g)$ be an object in $\Lambda(X)$. By $[\mathbf{2 3}, 4.1 .1]$, we may identify $(L V)_{(x, g)}$ with the space of loops

$$
\left\{\gamma: \mathbb{R} \rightarrow V_{x} \mid \gamma(t+1)=g \gamma(t)\right\} .
$$

So,

$$
L V_{(x, g)} \subseteq \operatorname{maps}\left(\mathbb{R} /|g| \mathbb{Z}, V_{x}\right) .
$$

The Fourier expansion principle gives a dense isomorphism

$$
\operatorname{maps}\left(\mathbb{R} /|g| \mathbb{Z}, V_{x}\right) \cong V_{x, 0} \oplus \bigoplus_{n \geqslant 1} V_{x}^{\mathbb{C}} q^{\frac{n}{|g|}}
$$

under which $L V_{(x, g)}$ maps to the submodule specified in the lemma.

\subsubsection{Moonshine}

Let $M$ be the monster, and consider the central extension $\widetilde{\Lambda}_{\alpha}(\mathrm{pt} / / M)$ of the inertia groupoid $\Lambda(\mathrm{pt} / / M)$ classified by the Moonshine cocycle

$$
\alpha \in H^{3}(B M, \mathrm{U}(1))
$$

(see [26, Meta Thm. p. 29]). Let $\widetilde{\xi}$ be a lift of $\xi^{1}$ to an element of the center of $\widetilde{\Lambda}_{\alpha}(\mathrm{pt} / / M)$. Then

$$
\widetilde{\Lambda}_{\alpha}(\mathrm{pt} / / M) \simeq \coprod_{[g]} \mathrm{pt} / / \widetilde{C}_{g}
$$

where the $\widetilde{C}_{g}$ are central extensions of the centralizers in $M$. A choice of $\widetilde{\xi}$ amounts to a choice of lift $\widetilde{g} \in \widetilde{C}_{g}$ for each $g$ in a system of representatives for the conjugacy classes of $M$. A Laurent series

$$
F \in \bigoplus_{n \geqslant 0} \operatorname{Rep}\left(\widetilde{C}_{g}\right)\left(\left(q^{\frac{1}{|\widetilde{g}|}}\right)\right)
$$

satisfies the rotation condition if and only if its character, a function $F\left(g, h ; q^{\frac{1}{|\widetilde{g}|}}\right)$ in $h \in \widetilde{C}_{g}$, satisfies

$$
F\left(g, \widetilde{g} h ; q^{\frac{1}{|\widetilde{g}|}}\right)=F\left(g, h ; e^{\frac{2 \pi i}{|\widetilde{g}|}} q^{\frac{1}{|\widetilde{g}|}}\right) .
$$

Interpreting the coefficients as characters of projective representations of $C_{g}$, this equation becomes

$$
F(g, g h ; \tau)=\zeta \cdot F(g, h ; \tau+1),
$$

where $\zeta$ is a root of unity and $q=e^{2 \pi i \tau}$. These are Condition (3) and half of Condition (1) of Norton's generalized Moonshine conjecture [30].

\subsection{Properties}

Tate $K$-theory is an orbifold theory with transfers. In other words, it satisfies the following list of properties: 
Orbifold theory. Let

$$
f:(X, \xi) \longrightarrow(Y, v)
$$

be a 1-morphisms in $\operatorname{Gpd}^{\mathrm{cen}}$. Then $f$ induces a ring map

$$
f^{*}: K_{\text {rot }}(Y, v) \longrightarrow K_{\text {rot }}(X, \xi) .
$$

If $f$ and $g$ are naturally isomorphic then $f^{*}=g^{*}$, and if $f$ is an equivalence of groupoids then $f^{*}$ is an isomorphism. It follows that the analogous statements hold for $K_{\text {Tate }}$, and that the latter is a well-defined functor on Moerdijk's orbifold category.

Sums and products. We have natural isomorphisms

$$
\begin{aligned}
K_{\text {Tate }}(\emptyset) & \cong\{0\}, \\
K_{\text {Tate }}(\mathrm{pt}) & \cong \mathbb{Z} \llbracket q \rrbracket, \\
K_{\text {Tate }}(X \sqcup Y) & \cong K_{\text {Tate }}(X) \oplus K_{\text {Tate }}(Y),
\end{aligned}
$$

and natural maps

$$
K_{\text {Tate }}(X) \otimes K_{\text {Tate }}(Y) \longrightarrow K_{\text {Tate }}(X \times Y),
$$

making $K_{\text {Tate }}$ a lax rig functor from Gpd to abelian rings.

Exactness. Fix a finite group $G$. Then the functor

$$
M \longmapsto K_{\text {Tate }}(M / / G)
$$

sends cofiber sequences of $G$-spaces to exact sequences.

Proof. Indeed, for any element $g \in G$, the functor

$$
\begin{aligned}
G \text {-spaces } & \longrightarrow C_{g^{\text {-spaces }}} \\
X & \longmapsto X^{g}
\end{aligned}
$$

preserves cofiber sequences.

\subsection{Transfers}

In this section all groupoids are assumed to have finite stabilizer groups. Given two maps of groupoids

$$
X \stackrel{u}{\longrightarrow} Z \stackrel{a}{\longleftarrow} Y
$$

we can form the fiber square

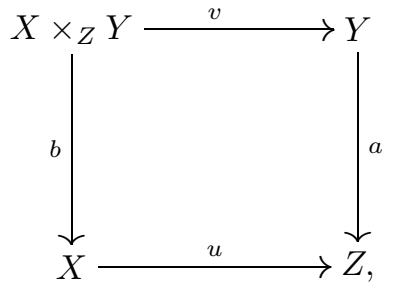

as in $[\mathbf{2 8}, 2.3]$, commuting up to a natural isomorphism $\eta$ (part of the data) and universal with respect to this property. We will refer to the groupoid $X \times_{Z} Y$ as the 
fibred product of $X$ and $Y$ over $Z$. Its objects are triples $(x, y, u(x) \stackrel{g}{\rightarrow} a(y))$, consisting of an object of each $X$ and $Y$ and an arrow between their images in $Z$. Arrows between two such triples $(x, y, g)$ and $\left(x^{\prime}, y^{\prime}, g^{\prime}\right)$ are pairs of arrows $\left(x \stackrel{h}{\rightarrow} x^{\prime}, y \stackrel{k}{\rightarrow} y^{\prime}\right)$ in $X$ and $Y$ satisfying $g^{\prime} u(h)=a(k) g$. Examples are the comma category groupoids $y \uparrow f$ and $Y \uparrow f$, defined by the fiber squares
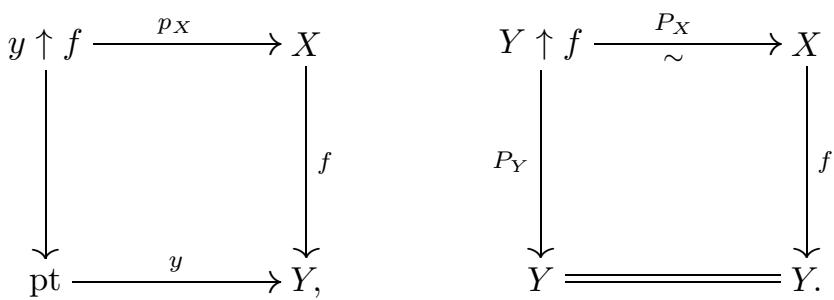

Definition 2.11. We say that $f: X \rightarrow Y$ is essentially a finite cover if for each object $y \in Y$, there is a neighborhood $U$ of $y$ in obY such that

$$
U \times_{Y} X \simeq U \times(y \uparrow f),
$$

and $y \uparrow f$ is equivalent to a finite groupoid. Here we have viewed $U$ as a groupoid with only identity morphisms, so that we can make sense of $U \times_{Y} X$ as in (5).

This condition ensures that pull-back of vector bundles possesses a right-adjoint

$$
\operatorname{RKan}_{f}: \operatorname{Vect}_{\mathbb{C}}(X) \longrightarrow \operatorname{Vect}_{\mathbb{C}}(Y),
$$

the right Kan extension along $f$, with

$$
\left(\operatorname{RKan}_{f} V\right)_{y}=\lim _{y \uparrow f}\left(p_{X}^{*} V\right) .
$$

Definition 2.12. Let $f: X \rightarrow Y$ be essentially a finite cover. Then we write

$$
f_{!}: K_{\text {orb }}(X) \longrightarrow K_{\text {orb }}(Y)
$$

For the map induced by $\operatorname{RKan}_{f}$. We will refer to $f_{!}$as the transfer along $f$.

Lemma 2.13. Let $f:(X, \xi) \longrightarrow(Y, v)$ be a 1-morphism in $\mathrm{Gpd}^{\mathrm{cen}}$. If $\xi$ acts with eigenvalue $e^{2 \pi i a}$ on the vector bundle $V$ on $X$, then $v$ acts with the same eigenvalue on $\operatorname{RKan}_{f} V$.

Proof. The action of $v$ on

$$
\left(\operatorname{RKan}_{f} V\right)_{y} \subseteq \bigoplus_{(x, g)} V_{x}
$$

is by the permutation of the summands sending the object $(x, g)$ of $y \uparrow f$ to $\left(x, v_{y} g\right)$. The limit condition applied to the arrow $\xi_{x}:\left(x, v_{y} g\right) \rightarrow(x, g)$ in $y \uparrow f$ forces an element of the limit to satisfy

$$
v_{(x, g)}=\xi_{x}\left(v_{\left(x, v_{y} g\right)}\right)=e^{2 \pi i a} \cdot v_{\left(x, v_{y} g\right)} .
$$

This proves the claim.

Corollary 2.14. We have transfers along essentially finite covers in $K_{\mathrm{rot}}$ and in $K_{\text {Tate }}$. 
Proof. The statement follows immediately from the fact that $\Lambda$ preserves essentially finite covers.

We will denote these transfers also by $f_{!}$. So, $f_{!}$in $K_{\text {Tate }}$ stands for $(\Lambda f)_{\text {! }}$ in $K_{\text {rot }}$.

Proposition 2.15. Given a fiber square as in (5), assume that the map a is essentially a finite cover. Then $b_{\text {! }}$ is defined, and we have

$$
u^{*} a_{!}=b_{!} v^{*} .
$$

Proof. Let $x$ be an object of $X$. Then we have an equivalence of groupoids

$$
\begin{aligned}
x \uparrow b & =\text { pt } \times_{X}\left(X \times_{Z} Y\right) \\
& \simeq \text { pt } \times_{Z} Y \\
& =u(x) \uparrow a .
\end{aligned}
$$

Consider the canonical natural transformation of functors $\operatorname{Vect}_{\mathbb{C}} Y \rightarrow \operatorname{Vect}_{\mathbb{C}} X$

$$
u^{*} \operatorname{RKan}_{a} \Longrightarrow \operatorname{RKan}_{b} v^{*} \text {. }
$$

Restricted to the fibers, this is the composition of isomorphisms

$$
\begin{aligned}
\left(\operatorname{RKan}_{b} v^{*} V\right)_{x} & =\lim _{\longleftarrow \uparrow b}\left(p_{\left(X \times_{Z} Y\right)}^{*} v^{*} V\right) \\
& \cong \lim _{\longleftarrow} u(x) \uparrow a \\
& =\left(p_{Y}^{*} V\right) \\
& \left.\operatorname{RKan}_{a}\right)_{u(x)} .
\end{aligned}
$$

Further, $\Lambda$ preserves fiber squares.

Corollary 2.16. Orbifold Tate K-theory, restricted to the 2 -category $\mathrm{Gpd}^{\text {fin }}$ of finite groupoids, is a global Mackey functor.

Here we are using the definition of global Mackey functor spelled out in [18]. It is well known that, in the same manner, orbifold $K$-theory gives the Mackey functor sending a finite group(oid) to its representation ring.

\subsection{Characters}

Unlike most of this paper, this section only applies to finite groupoids.

2.4.1. $n$-class functions

Let $X$ be a finite groupoid, let $R$ be a ring, and let $n \geqslant 0$ be a natural number.

Definition 2.17. An $n$-class function on $X$ with values in $R$ is an $R$-valued map

$$
\chi:\left[\Lambda^{n} X\right] \longrightarrow R,
$$

defined on the set of isomorphism classes of the $n$-fold inertia groupoid of $X$. We denote the ring of all such maps by

$$
n-\operatorname{class}(X, R) \cong H^{0}\left(\operatorname{Borel}\left(\Lambda^{n} X\right) ; R\right) .
$$


Explicitly, a 0-class function is a function on the isomorphism classes of $X$, and for $n \geqslant 1$, an $n$-class function is defined on $n$-tuples of commuting automorphisms of $X$ and satisfies

$$
\chi\left(g_{1}, \ldots, g_{n}\right)=\chi\left(s g_{1} s^{-1}, \ldots, s g_{n} s^{-1}\right) .
$$

Here all the $g_{i}$ are automorphisms of the same object $x$. It is well known that 0 -class $(-, R)$ is a global Mackey functor, namely group cohomology, with transfers along faithful maps $f: X \rightarrow Y$. It follows that $n$-class $(-, R)$ is also a Mackey functor, whose transfers along faithful maps are given by

$$
f_{!}(\chi)\left(g_{1}, \ldots, g_{n}\right)=\sum_{[\underline{h}] \mapsto[\underline{g}]} \frac{|\operatorname{aut}(\underline{g})|}{|\operatorname{aut}(\underline{h})|} \cdot \chi\left(h_{1}, \ldots, h_{n}\right) .
$$

Here $g$ abbreviates $\left(g_{1}, \ldots, g_{n}\right)$, and $[g]$ and aut $(g)$ are, respectively, its isomorphism class and its automorphism group in $\Lambda^{n} Y$, and similarly for $\underline{h}$.

If $R$ is a $\mathbb{Q}$-algebra, then (6) makes sense for all $f$ and extends $n$-class $(-; R)$ to a Mackey functor with all transfers.

Example 2.18. Let $j: H \rightarrow G$ be an inclusion of groups and write $j$ also for the corresponding map of groupoids $j: \mathrm{pt} / / H \rightarrow \mathrm{pt} / / G$. Then

$$
j_{!}(\chi)\left(g_{1}, \ldots, g_{n}\right)=\frac{1}{|H|} \sum_{s \underline{g} s^{-1} \in H^{n}} \chi\left(s g_{1} s^{-1}, \ldots, s g_{n} s^{-1}\right) .
$$

Example 2.19. Let $G$ be a finite group, and let $\varepsilon_{G}: \mathrm{pt} / / G \rightarrow$ pt be the unique map. Then

$$
\left(\varepsilon_{G}\right) !(\chi)(1)=\frac{1}{|G|} \sum_{\underline{g}} \chi\left(g_{1}, \ldots, g_{n}\right),
$$

where the sum runs over all $n$-tuples of commuting elements of $G$.

Note that, unlike equation (6), the sums in these examples are over elements, rather than conjugacy classes. This is consistent, because various $\mid$ aut(.)| factors cancel out.

\subsubsection{Character theory}

Write $\mathrm{Gpd}^{f}$ for the 2-category of finite groupoids, and assume that $E$ is an orbifold theory with transfers as in Section 2.2 .

Definition 2.20. A character theory for $E$ of height $n$ consists of a ring $R$ and a natural transformation of Mackey functors

$$
\chi:\left.E\right|_{\mathrm{Gpd}^{f}} \Longrightarrow n \text {-class }(-, R) .
$$

Here $R$ is assumed to be either a $\mathbb{Q}$-algebra or torsion free with the understanding that some transfers in $n$-class $(-, R)$ are, a priori, only defined over $\mathbb{Q} \otimes R$. Naturality of $\chi$ then implies integrality of transfers in the image of $\chi$.

Example 2.21. $K$-theory has a character theory of height 1 with

$$
R=\underline{\lim }_{k} \mathbb{Z}\left[\zeta_{k}\right],
$$

where $\zeta_{k}$ is a $k$ th root of 1 . Note that the universal level $k$-structure on the multiplicative group has its home over $\operatorname{spec}\left(\mathbb{Z}\left[\zeta_{k}\right]\right)$. The integrality statement above amounts 
to the well-known fact that

$$
\frac{1}{|G|} \sum_{g \in G} \chi(g) \in \mathbb{Z}
$$

when $\chi$ is the character of a representation.

Example 2.22. Tate $K$-theory has a height 2 character theory, taking values in

$$
\lim _{\longrightarrow k}\left(\mathbb{Z}\left[\zeta_{k}\right] \llbracket q^{\frac{1}{k}} \rrbracket\right) ;
$$

compare to Section 2.1.1. By [13, VII.2.4,VII.1.16.4]),

$$
\operatorname{spec} \mathbb{Z}\left[\zeta_{k}\right] \llbracket q^{\frac{1}{k}} \rrbracket \longrightarrow\left(\mathcal{M}_{k}\right)
$$

is the completion at infinity of the level $k$ stack $\mathcal{M}_{k}$ defined in [13, p. 36, IV.3.2,3.5].

Example 2.23. Fix a prime $p$, and let $E_{n}$ be Borel equivariant Morava-Lubin-Tate theory. This has a height $n$ character theory $([\mathbf{1 6}, 7.9],[\mathbf{2 2}$, Thms. C\&D]), taking values in

$$
L\left(E_{n}\right)=S^{-1} \underline{\lim }_{k} E_{n}\left(B\left(\mathbb{Z} / p^{k} \mathbb{Z}\right)^{n}\right),
$$

where the multiplicative set $S$ consists of the Euler classes of non-trivial representations. The ring

$$
D_{k}:=\operatorname{im}\left(E_{n}\left(B\left(\mathbb{Z} / p^{k} \mathbb{Z}\right)^{n}\right) \rightarrow S^{-1} E_{n}\left(B\left(\mathbb{Z} / p^{k} \mathbb{Z}\right)^{n}\right)\right)
$$

is the home of the universal level $k$-structure on the formal group of $E_{n}$ (see [1, Thm. 3.3.2]).

The character theory in $[\mathbf{2 2}]$ is a lot more powerful than our summary here suggests. It would be interesting to formulate Hopkins-Kuhn-Ravenel theory for orbifolds and to see exactly how much of the Hopkins-Kuhn-Ravenel story can be told for $K_{\text {Tate }}$. It seems reasonable to expect that $K_{\text {Tate }}$ fits into Stapleton's framework of transchromatic character maps [33].

2.4.3. A formula for induction

Let $H \subseteq G$ be an inclusion of finite groups, $M$ a $G$-manifold, and let

$$
j: M / / H \longrightarrow M / / G
$$

be the inclusion. Then the transfer $j$ ! in $K_{\text {orb }}$ is

$$
\operatorname{ind}_{H}^{G}: K_{H}(M) \longrightarrow K_{G}(M) \text {. }
$$

We will write

$$
I_{H}^{G}: K_{\text {Tate }}(M / / H) \longrightarrow K_{\text {Tate }}(M / / G)
$$

for the transfer $j$ ! in $K_{\text {Tate }}$. Let $a$ be an element of $K_{\text {Tate }}(M / / H)$. Then $I_{H}^{G}(a)$ is an element of

$$
\bigoplus_{[g]} K_{C_{G}(g)}\left(M^{g}\right) \llbracket q^{\frac{1}{|g|} \rrbracket} .
$$

Fix $g \in G$, choose representatives $h_{1}, \ldots, h_{k}$ for the $H$-conjugacy classes contained in $[g]_{G}$, and for $1 \leqslant i \leqslant k$, choose $s_{i} \in G$ with $s_{i} h_{i} s_{i}^{-1}=g$. Write $C_{i}:=C_{H}\left(h_{i}\right)$, and 
$C_{G}:=C_{G}(g)$, and view the former as a subgroup of the latter via the inclusion

$$
\begin{aligned}
C_{i} & \hookrightarrow C_{G} \\
t & \mapsto s_{i} t s_{i}^{-1} .
\end{aligned}
$$

Proposition 2.24. With the above notation, the $[g]$ th summand of $I_{H}^{G}(a)$ equals

$$
I_{H}^{G}(a)_{[g]}=\bigoplus_{i=1}^{k} \operatorname{ind}_{C_{i}}^{C_{G}}\left(a_{i}\right),
$$

where $a_{i}=a_{\left[h_{i}\right]}$

Proof. Because of the commuting diagram

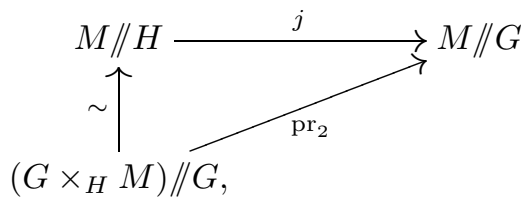

$I_{H}^{G}$ may be identified with the $G$-equivariant (Atiyah) transfer in $K_{\text {rot }}$ along $\Lambda\left(\operatorname{pr}_{2}\right)$. This may be viewed as the map of $G$-spaces

$$
\begin{aligned}
G \times_{H} \mathrm{ob}(\Lambda(M / / H)) & \longrightarrow \mathrm{ob}(\Lambda(M / / G) \\
(s,(x, h)) & \longmapsto\left(s x, s h s^{-1}\right) .
\end{aligned}
$$

Hence we have

$$
I_{H}^{G}(a)_{[g]}=\sum_{r^{-1} g r \in H} r \cdot a_{\left[r^{-1} g r\right]},
$$

where the sum runs over $r \in \mathcal{R}$, a system of representatives of $G / H$. Without loss of generality, the set of those representatives contributing to the sum may be chosen of the form

$$
s_{1} \cdot \mathcal{R}_{1} \sqcup \cdots \sqcup s_{k} \cdot \mathcal{R}_{k},
$$

with $\mathcal{R}_{i}$ a system of representatives for $C_{G}\left(h_{i}\right) / C_{H}\left(h_{i}\right)$. Then (7) becomes

$$
\bigoplus_{i=1}^{k} s_{i} \cdot \bigoplus_{r \in \mathcal{R}_{i}} r a_{\left[h_{i}\right]}=\bigoplus_{i=1}^{k} s_{i} \cdot \operatorname{ind}_{C_{H}\left(h_{i}\right)}^{C_{G}\left(h_{i}\right)} a_{\left[h_{i}\right]},
$$

as claimed.

\section{Power operations}

\subsection{Symmetric powers of orbifolds}

Recall that Gpd is a symmetric bimonoidal category ("rig" category) with the monoidal structures given by $(\sqcup, \emptyset)$ and $(\times, \mathrm{pt})$. Every groupoid $X \in \mathrm{ob}(\mathrm{Gpd})$ is a monoid with respect to $(\sqcup, \emptyset)$, via the fold map

$$
d: X \sqcup X \longrightarrow X .
$$

A groupoid $X$ is a monoid with respect to $(\times, p t)$ if and only if $X$ is endowed with the structure of a symmetric monoidal category. 
Definition 3.1. Then $n$th symmetric power of $X \in \mathrm{ob}(\mathrm{Gpd})$ is the groupoid $S_{n}$ \X obtained from $X^{n}$ by adding the additional arrows

$$
\left(x_{1}, \ldots, x_{n}\right) \stackrel{\sigma}{\longrightarrow}\left(x_{\sigma^{-1}(1)}, \ldots, x_{\sigma^{-1}(n)}\right) \quad \sigma \in S_{n},
$$

composing with the arrows in $X^{n}$ as follows:

$$
\sigma \circ\left(g_{1}, \ldots, g_{n}\right)=\left(g_{\sigma^{-1}(1)}, \ldots, g_{\sigma^{-1}(n)}\right) \circ \sigma .
$$

The total symmetric power of $X$ is

$$
S(X):=\coprod_{n \geqslant 0} S_{n} \curlywedge X .
$$

The endofunctor $S$ of $G p d$ is exponential in the sense that it is a monoidal functor

$$
S:(\mathrm{Gpd}, \sqcup, \emptyset) \longrightarrow(\mathrm{Gpd}, \times, \mathrm{pt}) .
$$

It follows that $S(X)$ comes equipped with a symmetric monoidal structure

$$
*:=S(d)
$$

which turns out to be concatenation. The unit of $*$ is the unique object () of $S_{0}$ 乙X. The triple

$$
(S(X), *,())
$$

may be viewed as the free symmetric monoidal category on $X$. More precisely, the functor

$$
X \longmapsto(S(X), *,())
$$

is the left adjoint to the forgetful functor from the category of monoids in Gpd to Gpd. In particular, $S$ is a monad.

Definition 3.2. We will write

$$
\mu: S^{2} \Longrightarrow S
$$

and

$$
\iota: \mathrm{Id} \Longrightarrow S
$$

for the structure maps of the monad $S$. Explicitly, these are given by the inclusions $S_{n} \prec S_{m} \subseteq S_{n m}$ and by the inclusion of $X=S_{1} \prec X$ inside $S(X)$.

\subsection{The inertia groupoid of $S(X)$}

Objects of $\Lambda(S(X))$ are given by quadruples $(n, \underline{x}, \underline{g}, \sigma)$, where $n \geqslant 0$ is a natural

number, $\underline{x} \in \mathrm{ob}\left(X^{n}\right)$, and $\sigma \in S_{n}$, while $g$ is a system of arrows $x_{i} \stackrel{g_{i}}{\longrightarrow} x_{\sigma(i)}$. Note that this notation is somewhat redundant, all the information is contained in the pair $(\sigma ; g)$. The inertia groupoid inherits a monoidal structure from $S(X)$, given by

$$
\left(\sigma ; g_{1}, \ldots, g_{n}\right) *\left(\tau ; h_{1}, \ldots, h_{m}\right)=\left(\sigma \sqcup \tau ; g_{1}, \ldots, g_{n}, h_{1}, \ldots, h_{m}\right) .
$$

An object $(\sigma, g)$ of $\Lambda(S(X))$ is indecomposable with respect to $*$ if and only if $n>0$ and $\sigma$ acts on $\{1, \ldots, n\}$ with a single orbit (i.e., $\sigma$ is a long cycle). Otherwise, the cycle decomposition of $\sigma$ yields a decomposition of $(\sigma, \underline{g})$ into indecomposables. 
Definition 3.3. Let $\varsigma_{k} \in S_{k}$ be the cycle $\varsigma_{k}=(1, \ldots, k)$, and let $\Phi_{k}(X)$ be the full subgroupoid of $\Lambda(S(X))$ with objects $\left(\varsigma_{k}, \underline{x}\right)$. Let $\Phi(X)$ be the groupoid

$$
\Phi(X):=\coprod_{k \geqslant 1} \Phi_{k}(X) .
$$

Further, let $\varphi \in \operatorname{Center}(\Phi)$ be the restriction of $\xi^{1}$ to $\Phi(X)$.

The essential image of $\Phi(X)$ inside $\Lambda(S X)$ is the subgroupoid of indecomposable objects. The functor $\Phi$ is additive, i.e., $\Phi(\emptyset)=\emptyset$, and $\Phi$ preserves $\sqcup$.

Lemma 3.4. We have an equivalence of monoidal groupoids

$$
Q: S(\Phi(X)) \longrightarrow \Lambda(S(X)),
$$

which is natural in $X$ and satisfies

$$
Q S(\varphi)=\xi^{1} Q
$$

Proof. Let $I$ be the inclusion

$$
I: \Phi(X) \hookrightarrow \Lambda(S(X))
$$

and let $Q$ be the composite

$$
Q: S(\Phi(X)) \stackrel{S(I)}{\longrightarrow} S(\Lambda(S(X))) \stackrel{\varepsilon}{\longrightarrow} \Lambda(S(X)),
$$

where the second map is the counit of the adjunction $(S, *,()) \dashv$ forget. Then $Q$ is monoidal. Since the essential image of $I$ consists exactly of the indecomposable objects of $\Lambda(S(X))$, it follows that $Q$ is essentially surjective. One checks that $Q$ is also fully faithful. (This boils down to the fact that $\sigma$ and $\tau$ are conjugate in $S_{n}$ if and only if their cycle decompositions are congruent.)

Definition 3.5. For each $k \geqslant 1$, we define a map of groupoids

$$
\begin{aligned}
E_{k}: \Phi_{k}(X) & \longrightarrow \Lambda(X)\left[\xi^{\frac{1}{k}}\right] & & \\
\left(\left(x_{1}, \ldots, x_{k}\right),\left(\varsigma_{k} ; g_{1}, \ldots, g_{k}\right)\right) & \longmapsto\left(x_{1}, g_{k} \cdots g_{1}\right) & & \text { on objects, and } \\
\left.\left(\mathrm{id} ; h_{1}, \ldots, h_{k}\right)\right) & \longmapsto h_{1} & & \text { and } \\
\varphi & \longmapsto \xi^{\frac{1}{k}} & & \text { on morphisms. }
\end{aligned}
$$

Lemma 3.6. The map $E_{k}$ is a well-defined equivalence of groupoids.

In order to make the notation more manageable, we will simply write $g$ for the object of $\Lambda(X)$ previously denoted $(x, g)$. 
Proof. It is clear that $E_{k}$ is surjective on objects. Let $\left(\varsigma_{k} ; g\right)$ be an object of $\Phi_{k}(X)$, and write (id; $\underline{\widehat{g}}$ ) for its image under $\Pi^{k}$. In other words, $\widehat{g}_{i} \in \mathrm{ob}(\Lambda(X))$ is the composite

$$
\widehat{g}_{i}: x_{i} \stackrel{g_{i}}{\longrightarrow} x_{i+1} \stackrel{g_{i+1}}{\longrightarrow} x_{i+2} \stackrel{g_{i+2}}{\longrightarrow} \cdots x_{i-1} \stackrel{g_{i-1}}{\longrightarrow} x_{i} .
$$

Then we have

$$
\varphi_{(\underline{x}, \underline{g})}^{k}=(\mathrm{id}, \underline{\widehat{g}}) .
$$

Let $\left(\varsigma_{k}^{m}, \underline{h}\right)$ be an arrow in $\Phi_{k}(X)$. Then $\left(\varsigma_{k}^{m}, \underline{h}\right)$ can be factored as

$$
\left(\mathrm{id}, \underline{h}^{\prime}\right) \circ \varphi^{m}=\varphi^{m} \circ\left(\mathrm{id}, \underline{h}^{\prime}\right)
$$

with $m \in \mathbb{Z}$, and this presentation is unique up to the relation (8).

Consider an arrow of the form $(\mathrm{id}, \underline{h})$ from $(\underline{x}, \underline{g})$ to $\left(\underline{x}^{\prime}, \underline{g}^{\prime}\right)$ in $\Phi_{k}(X)$. Then the $h_{i}$ fit into a commuting diagram

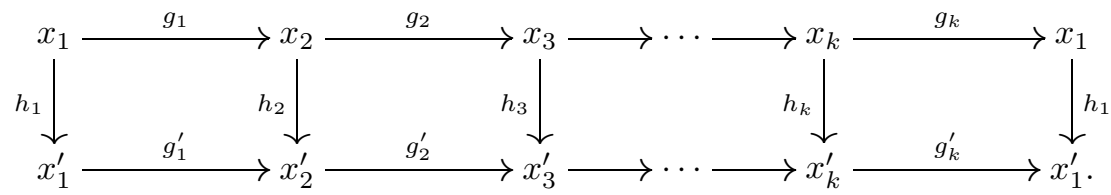

In particular,

$$
h_{1} \widehat{g}_{1} h_{1}^{-1}=\widehat{g}_{1}^{\prime}
$$

(and, similarly for $i$ ). Hence $E_{k}$ is well defined. Further, the $h_{i}$ are uniquely determined by $h_{1}$, and any $h_{1}$ satisfying (10) gives rise a to system $\underline{h}$ fitting into (9). Hence $E_{k}$ is fully faithful.

Definition 3.7. Let $F_{k}$ be the quasi-inverse of $E_{k}$ that sends the object $g$ to

$$
\left(\varsigma_{k} ; g, \mathrm{id}, \ldots, \mathrm{id}\right)
$$

$(k-1$ times id).

All the above constructions are Morita invariant.

3.2.1. Comparison to Dijkgraaf, Moore, Verlinde and Verlinde

Let $X$ be the groupoid of a global quotient orbifold $M / / G$, where $G$ is a finite group. Then the loop space of $S_{n} \prec X$,

$$
L\left(S_{n} \curlywedge X\right) \simeq \operatorname{Fun}\left(\mathbb{R} / / \mathbb{Z}, S_{n} \curlywedge X\right),
$$

has objects $(\sigma ; \underline{g}, \underline{\gamma})$, where $(\sigma ; \underline{g}) \in S_{n} \prec G$ and $\underline{\gamma}$ is an $n$-tuple of paths

$$
\gamma_{i}: \mathbb{R} \longrightarrow M
$$

satisfying

$$
g_{i} \gamma_{i}(t)=\gamma_{\sigma(i)}(t+1)
$$


Example 3.8. Assume that $\sigma=\varsigma_{k}$. Then we have

$$
\begin{aligned}
& \gamma_{1}(t+k)=g_{k} \cdots g_{1} \gamma_{1}(t) \\
& \gamma_{2}(t+k)=g_{1} g_{k} \cdots g_{2} \gamma_{2}(t)
\end{aligned}
$$

and so on, and each $\gamma_{i}$ determines the others via (11). Hence

$$
\gamma_{i} \in \operatorname{Fun}(\mathbb{R} / / k \mathbb{Z}, X)
$$

may be thought of as a loop of length $k$ in $M / / G$.

Let $L_{k} X \subseteq L\left(S_{n} \curlywedge X\right)$ be the full subgroupoid whose objects have $\sigma=\varsigma_{k}$. Then our groupoid $\Phi_{k}$ can be identified with $L_{k} X^{\mathbb{R}}$, the part of $L_{k} X$ that is invariant under the rotation action by $\mathbb{R}$. So, $\Phi_{k} X$ may be viewed as the groupoid of constant loops of length $k$ in $M / / G$, and $\varphi$ is identified with the rotation action by $1 \in \mathbb{Z}$ on these long loops. The key argument in $[\mathbf{1 5}]$ is summarized by the equivalence of groupoids

$$
L S(X) \simeq S\left(\coprod_{k \geqslant 1} L_{k} X\right)
$$

(see also [34]). Our Lemma 3.4 above follows from (12) by restricting to constant loops on both sides.

\subsection{Power operations in orbifold theories}

Let $E$ be an orbifold theory with products.

Definition 3.9. A total power operation for $E$ is a (non-linear) natural transformation

$$
P: E \Longrightarrow E \circ S
$$

satisfying the following properties:

Comodule property. $P$ makes $E$ a comodule over the comonad (-) $\circ S$, i.e., the following diagrams commute:
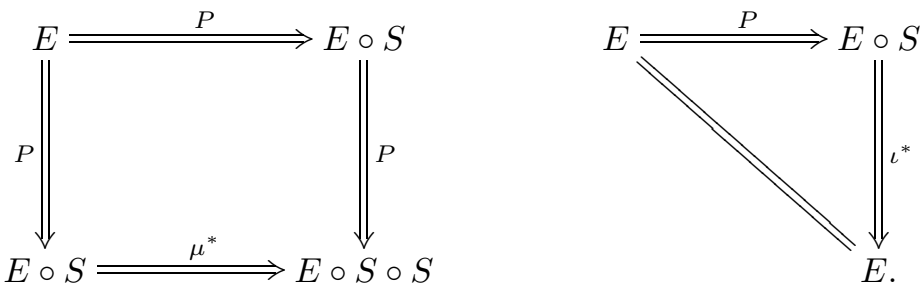

Exponentiality: The map

$$
P: E(\emptyset) \longrightarrow E(\mathrm{pt})
$$

sends 0 to 1 , and

$$
P: E(X \sqcup Y) \longrightarrow E(S X \times S Y)
$$

sends $(a, b)$ to the external product $P(a) \otimes P(b)$.

We can write $P$ as $P=\left(P_{n}\right)_{n \geqslant 0}$, with $P_{n}:=\iota_{n}^{*} P$. Then $P_{n}$ is called the $n$th power operation of $P$. 
Example 3.10 (Atiyah power operations). Let $V$ be an orbifold vector bundle on $X$. Then we have the Atiyah Power Operations

$$
P_{n}(V):=S_{n} \ltimes V^{\otimes n}
$$

of [6]. We will see below how to extend this definition to virtual vector bundles. For now we note that $P=\left(P_{n}\right)_{n \geqslant 0}$ satisfies the axioms of a total power operation, whenever it is defined.

3.3.1. Consequences of the definition

\section{Proposition 3.11.}

1. We have $P_{1}(a)=a$.

2. More generally,

$$
i_{k}^{*} P_{k}(a)=a^{\otimes k}
$$

(external product), where $i_{k}$ is the inclusion of $X^{k}$ in $S_{k} \prec X$.

3. We have

$$
(P S) \circ P=\mu^{*} \circ P,
$$

or, equivalently,

$$
P_{n}\left(P_{m}(a)\right)=\operatorname{res}_{S_{n} \backslash S_{m}}^{S_{n m}}\left(P_{n m}(a)\right) .
$$

4. $P_{0}(a)=1$.

5. The external product of $a \in E(X)$ and $b \in E(Y)$ is given by

$$
a \otimes b=i^{*} P(a, b),
$$

where $(a, b) \in E(X \sqcup Y)$, and

$$
i:=\left(\iota_{X} \times \iota_{Y}\right): X \times Y \hookrightarrow S X \times S Y
$$

is the product of the canonical inclusions (see Definition 3.2).

6. We have

$$
P(a \otimes b)=j^{*}(P(a) \otimes P(b))
$$

(external products), where

$$
j: S(X \times Y) \longrightarrow S(X) \times S(Y)
$$

is defined as

$$
j=\left(S\left(\pi_{X}\right), S\left(\pi_{Y}\right)\right),
$$

with $\pi_{X}$ and $\pi_{Y}$ the projections to the respective factors.

7. The map $P$, and equivalently all the $P_{n}$, preserves interior products:

$$
P_{n}(a b)=P_{n}(a) P_{n}(b),
$$

for $a, b \in E(X)$.

8. For each $n$, we have $P_{n}(1)=1$. 
Proof. The Comodule Property translates into Points 1. and 3. To prove Point 2., we apply naturality of $P$ to the fold map $f: X \sqcup \cdots \sqcup X \rightarrow X$, noting that $S(f)$ is the concatenation product $*$. This leads to a commuting diagram

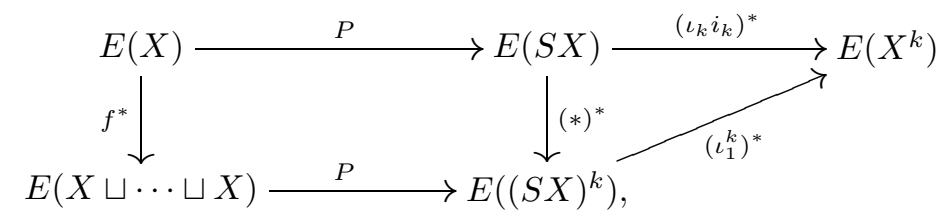

where $f^{*}(a)=(a, \ldots, a)$. By the Exponential Property, the bottom composite sends $(a, \ldots, a)$ to $P_{1}(a)^{\otimes k}$, hence 2 .

For Point 4., we apply naturality of $P$ to the unique map $e: \emptyset \rightarrow X$, arriving at

$$
\left.\iota_{0}^{*} \circ P\right|_{X}=\left.P\right|_{\emptyset} \circ e^{*} .
$$

The left-hand side of this is $P_{0}$. By the first part of the Exponential Property, the right-hand side is the constant map 1.

Point 5. is an immediate consequence of the Exponential Property and 1.

To prove Point 6 ., one checks that $j=\mu \circ S(i)$ and considers the diagram

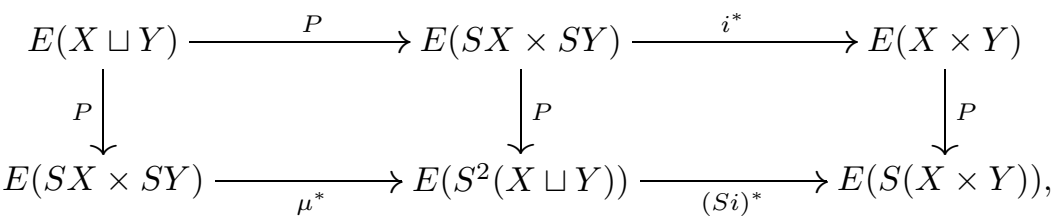

whose left square commutes by 3., and whose right square commutes by naturality of $P$. By Point 5 ., the composite on the top row sends $(a, b)$ to $a \otimes b$. By the Exponential Property, the leftmost vertical arrow sends $(a, b)$ to $P(a) \otimes P(b)$.

Point 7. follows from 6 . by considering the special case $X=Y$ : Let $\delta: X \rightarrow X \times X$ be the diagonal map. Then we have

$$
\begin{aligned}
P(a b) & =P\left(\delta^{*}(a \otimes b)\right) \\
& =(S \delta)^{*} P(a \otimes b) \\
& =(S \delta)^{*} j^{*}(P(a) \otimes P(b)) \\
& =\delta_{S(X)}^{*}(P(a) \otimes P(b)) \\
& =P(a) P(b) .
\end{aligned}
$$

Finally, we show Point 8 . In the special case $X=$ pt, this follows from 3 . and the first part of the Exponential Property. The general case follows by applying naturality of $P$ to the unique map from $X$ to pt.

\subsection{The graded ring $E(S(X))$}

From now on, we assume that $E$ possesses transfers along (faithful) essentially finite covers. Then transfer along the concatenation product

$$
*: S(X) \times S(X) \longrightarrow S(X)
$$


defines a second multiplication $\bullet$ on the ring $E(S(X))$, making it a graded ring with respect to the grading

$$
E(S(X)) \cong \bigoplus_{n \geqslant 0}^{\wedge} E\left(S_{n} \curlywedge X\right)
$$

with unit 1 in degree zero.

Proposition 3.12. Assume that $P$ is natural also with respect to transfers, and let $a, b \in E(X)$. Then we have

$$
P(0)=1
$$

and

$$
P(a+b)=P(a) \bullet P(b) .
$$

Proof. The first equality follows by applying naturality of $P$ to the transfer along the inclusion of $\emptyset$ in $X$. The second follows by applying naturality of $P$ to the transfer along the fold map $X \sqcup X \rightarrow X$.

Assume now that $E$ is an orbifold theory such that, for each finite group $G$, the functor $E_{G}(M)=E(M / / G)$ is not just exact, but actually the degree zero part of a $G$-equivariant cohomology theory. In this situation, we demand that $P$ be natural also with respect to maps in the $G$-equivariant stable category. Spanier-Whitehead duality implies that each $E_{G}$ possesses transfers along $G$-equivariant finite covers and our assumption on $P$ implies that $P$ is natural with respect to these transfers. Note that by the proof of Proposition 2.24, this class of transfers includes transfers along the maps

$$
M / / H \longrightarrow M / / G,
$$

induced by the inclusion of a subgroup $H \subseteq G$. In particular, $P$ is natural with respect to the map

$$
*: S X \times S X \rightarrow S X
$$

whenever $X / / G$ is a global quotient orbifold.

It seems reasonable to expect that, in a suitably defined category of 'orbispectra', a similar argument would make the demand for naturality along faithful transfers redundant and that all $K$-theoretic transfers above should be induced by maps in a

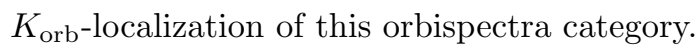

Corollary 3.13. The Atiyah power operations of Example 3.10 can be extended, in a unique way, to virtual vector bundles.

Proof. Indeed, $P$ takes values in $\mathbf{1}+K_{\mathrm{orb}}(S X)$ and hence $P([V])$ is invertible with respect to $\bullet$ for any vector bundle $V$.

Similarly, one argues that the definition of Atiyah power operations extends to $K_{\text {rot }}$, giving

$$
P: K_{\mathrm{rot}}(X, \xi) \longrightarrow K_{\mathrm{rot}}(S X, S \xi)
$$




\subsection{Power operations in Tate $K$-theory}

Recall from Lemma 3.4 that we have

$$
K_{\text {Tate }}(S(X)) \cong K_{\text {rot }}(S(\Phi(X)), S(\varphi)) .
$$

This becomes a graded isomorphism if, on the right-hand side, elements supported on $S_{n} \prec \Phi_{k}(X)$ are given degree $n k$.

Definition 3.14. Let $X$ be an orbifold groupoid, let $\xi$ be an element of its center, and let $k \geqslant 1$ be a natural number. Then we define the map

$$
\begin{aligned}
s_{k}: K_{\mathrm{rot}}(X) & \longrightarrow K_{\mathrm{rot}}\left(X\left[\xi^{\frac{1}{k}}\right]\right) \\
{\left[\sum V_{a} q^{a}\right] } & \longmapsto\left[\sum V_{a} q^{\frac{a}{k}}\right],
\end{aligned}
$$

where $\xi^{\frac{1}{k}}$ acts on the coefficient $V_{a}$ by $e^{2 \pi i a / k}$.

The map $s_{k}$ commutes with the Atiyah power operations in the following sense: let $(X, \xi)$ be as in Definition 3.14, recall the notation $X^{(k)}$ for $X\left[\xi^{\frac{1}{k}}\right]$ and let

$$
\delta:(S X)^{(k)} \hookrightarrow S\left(X^{(k)}\right)
$$

be the canonical inclusion (i.e., $\delta$ sends $(S \xi)^{\frac{1}{k}}$ to $S\left(\xi^{\frac{1}{k}}\right)$ ). Then we have

$$
s_{k} \circ P=\delta^{*} P s_{k} .
$$

Definition 3.15. Let

$$
\theta: K_{\text {Tate }}(X) \longrightarrow K_{\text {rot }}(\Phi(X), \varphi)
$$

be the additive operation whose $k$ th component is $E_{k}^{*} \circ s_{k}$. Here $E_{k}$ is the equivalence defined in Lemma 3.6.

Definition 3.16. The total power operation in $K_{\text {Tate }}$ is defined as the composite

$$
P^{\text {Tate }}: K_{\text {Tate }}(X) \stackrel{\theta}{\longrightarrow} K_{\text {rot }}(\Phi X, \varphi) \stackrel{P}{\longrightarrow} K_{\text {rot }}(S \Phi X, S \varphi) \stackrel{\left(Q^{*}\right)^{-1}}{\longrightarrow} K_{\text {Tate }}(S X) .
$$

Theorem 3.17. This $P^{\text {Tate }}$ satisfies the axioms of a total power operation.

Proof. The Exponential Property follows immediately from that of $P$ and from additivity of $\theta$,

$$
\begin{aligned}
\theta: K_{\text {Tate }}(X \sqcup Y) & \longrightarrow K_{\text {rot }}(\Phi X \sqcup \Phi Y) \\
(a, b) & \longmapsto(\theta a, \theta b) .
\end{aligned}
$$

The following lemma is the first step towards proving the Comodule Property:

Lemma 3.18. Let the natural transformation $\vartheta$ be defined as the composite

$$
\vartheta_{X}: \Phi S X \stackrel{I_{S X}}{\longrightarrow} \Lambda S^{2} X \stackrel{\Lambda \mu_{X}}{\longrightarrow} \Lambda S X .
$$

(Note that $\vartheta$ and $\theta$ denote two different things.) Then we have

$$
(\theta S) \circ P^{\text {Tate }}=\vartheta^{*} P^{\text {Tate }} .
$$


In other words, the diagram

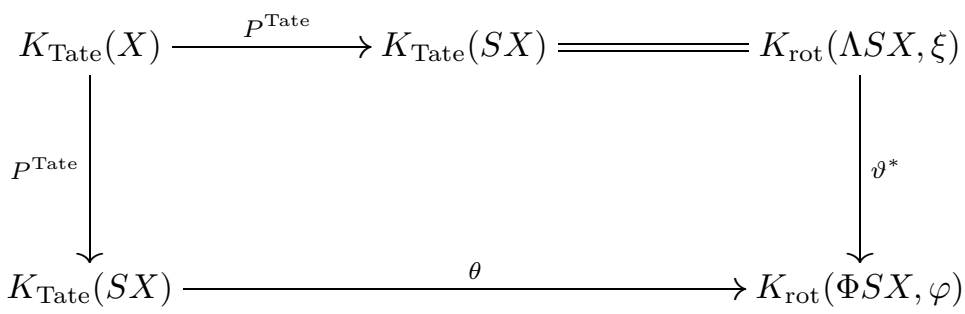

commutes.

Proof. It suffices to prove this equality after restricting both sides to $\Phi_{k} S$. Consider the commuting diagram

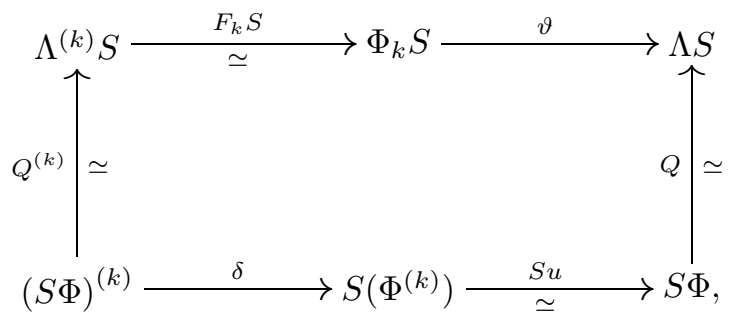

where the equivalence $u$ sends the object $\left(\varsigma_{l} ; \underline{g}\right)$ of $\left.\Phi_{l}^{(k)}\right)$ to the object

$$
(\varsigma_{k l} ; \underbrace{\mathrm{id}, \ldots, \mathrm{id}}_{k-1}, g_{1}, \underbrace{\mathrm{id}, \ldots, \mathrm{id}}_{k-1}, g_{2}, \mathrm{id}, \ldots, \ldots, \mathrm{id}, g_{l}),
$$

of $\Phi_{k l}$, sends a morphism of the form (id, $\left.\underline{h}\right)$ to

$$
(\mathrm{id} ; \underbrace{h_{1}, \ldots, h_{1}}_{k}, \underbrace{h_{2}, \ldots, h_{2}}_{k}, \ldots, \ldots, \underbrace{h_{l}, \ldots, h_{l}}_{k}),
$$

and $\varphi^{\frac{1}{k}}$ to $\varphi$. We have

$$
u^{*} \theta=s_{k} \theta
$$

and

$$
\left(Q^{(k)}\right)^{*} s_{k}=s_{k} Q^{*}
$$

where $s_{k}$ us as in Definition 3.14. Hence

$$
\begin{aligned}
\left(Q^{(k)}\right)^{*} \circ F_{k}^{*} \circ \vartheta^{*} \circ P^{\text {Tate }} & =\delta^{*} \circ(S u)^{*} \circ P \circ \theta \\
& =\delta^{*} \circ P \circ u^{*} \circ \theta \\
& =\delta^{*} \circ P \circ s_{k} \circ \theta \\
& =s_{k} \circ P \circ \theta \\
& =\left(Q^{(k)}\right)^{*} \circ s_{k} \circ P^{\text {Tate }} \\
& =\left(Q^{(k)}\right)^{*} \circ F_{k}^{*} \circ E_{k}^{*} \circ s_{k} \circ P^{\text {Tate }} \\
& =\left(Q^{(k)}\right)^{*} \circ F_{k}^{*} \circ \theta \circ s_{k} \circ P^{\text {Tate }}
\end{aligned}
$$

Since $\left(F_{k} \circ Q^{(k)}\right)^{*}$ is an isomorphism, the claim of the lemma follows. 
To complete the proof of the theorem, we consider the commuting diagram

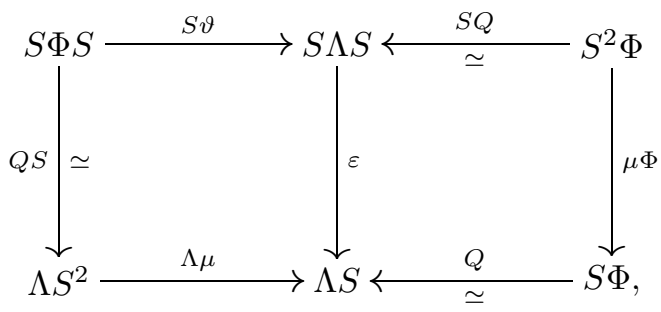

where the middle map, $\varepsilon$, is the counit of the adjunction $(S, *,()) \dashv$ forget (compare to the definition of $Q$ in the proof of Lemma 3.4). This yields

$$
\begin{aligned}
\left(P^{\text {Tate }} S\right) \circ P^{\text {Tate }} & =\left((Q S)^{*}\right)^{-1} \circ(P \Phi S) \circ \vartheta^{*} \circ\left(Q^{*}\right)^{-1} \circ(P \Phi) \circ \theta \\
& =\left((Q S)^{*}\right)^{-1} \circ(S \vartheta)^{*} \circ\left((S Q)^{*}\right)^{-1} \circ(P S \Phi) \circ(P \Phi) \circ \theta \\
& =\left((Q S)^{*}\right)^{-1} \circ(S \vartheta)^{*} \circ\left((S Q)^{*}\right)^{-1} \circ(\mu \Phi)^{*} \circ(P \Phi) \circ \theta \\
& =(\Lambda \mu)^{*}\left(Q^{*}\right)^{-1} \circ(P \Phi) \circ \theta \\
& =(\Lambda \mu)^{*} P^{\text {Tate }} .
\end{aligned}
$$

\subsection{Symmetric and exterior powers}

Let $E$ be a theory with transfers and power operations. Write

$$
\varepsilon: S(\mathrm{pt}) \longrightarrow \mathbb{N}
$$

for the augmentation map, sending $S_{n}$ 々 pt to $n$. We may identify $E(\mathbb{N} \times X)$ with the formal power series ring $E(X) \llbracket t \rrbracket$.

Definition 3.19. The total $E$-theoretic symmetric power $S_{t}$ is the composite

$$
S_{t}: E X \stackrel{P}{\longrightarrow} E S X \stackrel{\delta^{*}}{\longrightarrow} E(S(\mathrm{pt}) \times X) \stackrel{\varepsilon_{!}}{\longrightarrow} E(X) \llbracket t \rrbracket,
$$

where $\delta$ is the diagonal map, sending the arrow $(\sigma, g)$ to the arrow $(\sigma ; g, \ldots, g)$ with $n$ copies of $g$ if $\sigma \in S_{n}$.

Proposition 3.20. In the definition of $S_{t}$, the maps $\delta^{*}$ and $\varepsilon_{!}$are maps of graded rings.

Corollary 3.21. The total symmetric power is exponential:

$$
S_{t}(a+b)=S_{t}(a) \cdot S_{t}(b) .
$$

Proof. We now prove the proposition. The ring multiplication on $E(X) \llbracket t \rrbracket$ corresponds to

$$
E(\mathbb{N} \times X \times \mathbb{N} \times X) \stackrel{\delta_{X}^{*}}{\longrightarrow} E(\mathbb{N} \times X \times \mathbb{N}) \stackrel{m !}{\longrightarrow} E(\mathbb{N} \times X),
$$

where $\delta_{X}: X \rightarrow X \times X$ is the diagonal map and $m: \mathbb{N} \times \mathbb{N} \rightarrow \mathbb{N}$ is multiplication. Similarly, one defines the graded multiplication on $E(S(\mathrm{pt}) \times X)$ as the push-pull

$$
E\left((S(\mathrm{pt}) \times X)^{2}\right) \stackrel{\delta_{X}^{*}}{\longrightarrow} E\left(S(\mathrm{pt})^{2} \times X\right) \stackrel{*_{1}}{\longrightarrow} E(S(\mathrm{pt}) \times X) .
$$

The claim then follows by a tedious but straightforward iterated application of Proposition 2.15 . 


\subsection{Symmetric powers in Tate $K$-theory}

Definition 3.22. For a positive natural number $k$, we define the operator

$$
\begin{aligned}
\beta_{k}: K_{\text {Tate }}(X) & \longrightarrow K_{\text {Tate }}(X) \\
F & \longmapsto\left[\Pi_{k}^{*} F\left(q^{\frac{1}{k}}\right)\right]^{\mathbb{Z} / k \mathbb{Z}},
\end{aligned}
$$

where $\Pi_{k}$ is the $k$ th power map of Definition 2.5 , and the generator of $\mathbb{Z} / k \mathbb{Z}$ acts on the coefficient $\Pi_{k}^{*}\left(V_{a}\right)$ of $q^{\frac{a}{k}}$ by $\xi^{1} \cdot e^{-2 \pi i \frac{a}{k}}$. So, the $\mathbb{Z} / k \mathbb{Z}$-invariant part consists of the largest possible subspaces of the coefficients such that the result satisfies the rotation condition for $\xi^{1}$.

The action of the $\beta_{k}$ should be compared to the action of $\widehat{\mathbb{Z}}^{\times}$in $[\mathbf{2 2}]$.

Say the order of $\xi^{1}$ is $r$ and we are given an element $F\left(q^{\frac{1}{r}}\right) \in K_{\text {Tate }}(X)$. A priori, it looks like $\beta_{k}(F)$ is a power series in $q^{\frac{1}{k r}}$, but the rotation condition implies that it is in fact a power series in $q^{\frac{1}{r}}$.

Example 3.23. If $X=M$ is a manifold, then $F$ is of the form $F=\sum_{n=0}^{\infty} V_{n} q^{n}$, and

$$
\beta_{k}(F)=\sum_{m=1}^{\infty} V_{k m} q^{m}
$$

This should be compared to [15, (2.13)].

Theorem 3.24. The total symmetric power in Tate K-theory is described by the formula

$$
S_{t}^{\text {Tate }}(F)=\bigotimes_{k=1}^{\infty} S_{t^{k}}\left(\beta_{k}(F)\right) .
$$

Here $S_{t}$ stands for the total Atiyah symmetric power on $K_{\text {Tate }}$.

Corollary 3.25. If $X=M$ is a manifold and $F=V$ is a vector bundle on $M$, then $S_{t}^{\text {Tate }}(V)$ is Witten's stable exponential characteristic class,

$$
S_{t}^{\text {Tate }}(V)=\bigotimes_{k=1}^{\infty} S_{t^{k}}(V)
$$


Proof of Theorem 3.24. We will construct a commuting diagram:

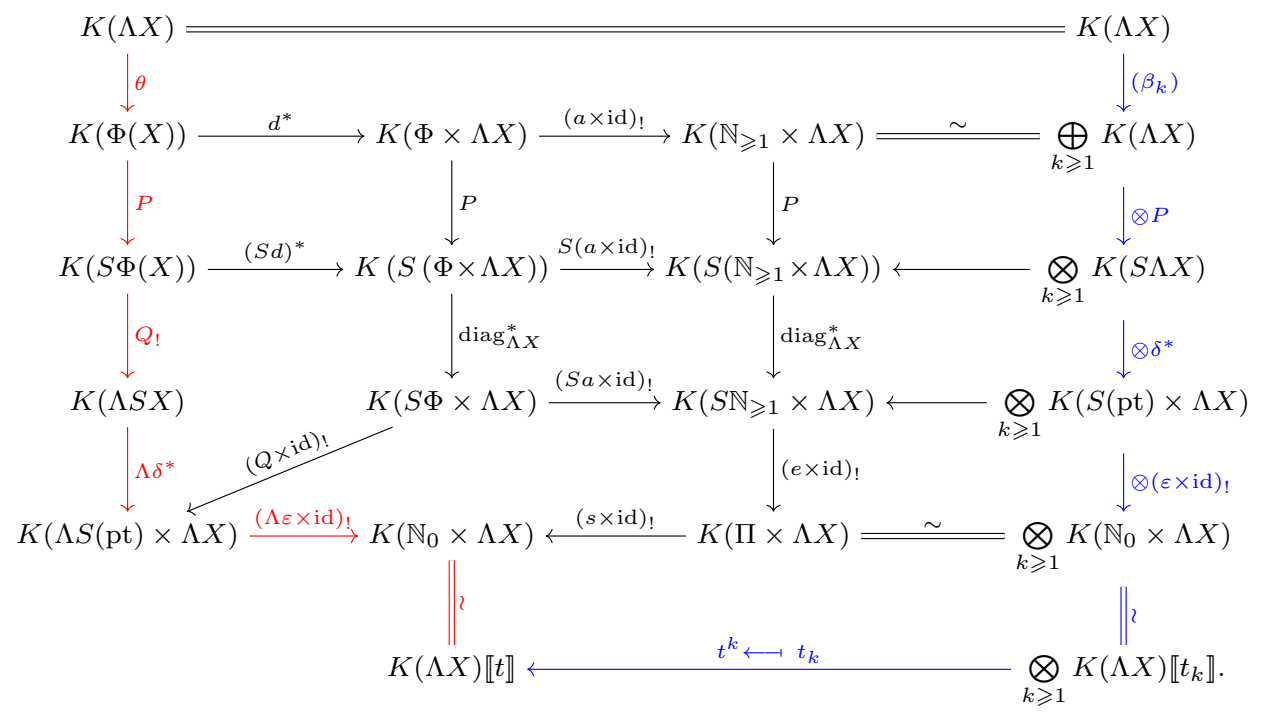

Again, $K$ is short for $K_{\text {rot }}$. The composite of the six red arrows (along the left edge of the diagram, starting in the upper-left corner and ending at $K(\Lambda X) \llbracket t \rrbracket)$ gives the left-hand side of (13), that of the six blue arrows (the composite of the right-most vertical arrows and the bottom arrow) gives the right-hand side. The other arrows are defined as follows: abbreviating $\Phi(\mathrm{pt})$ as $\Phi$, we let

$$
d: \Phi \times \Lambda X \rightarrow \Phi(X)
$$

be the restriction of $\Lambda \delta$ to the source of $d$. So, the $k$ th component $d_{k}$ of $d$ sends the object $g$ to $\left(\varsigma_{k} ; g, \ldots, g\right)$ and the morphism $\left(\varsigma_{k}^{m}, h\right)$ to $\left(\varsigma_{k}^{m} ; h, \ldots, h\right)$. Let

$$
a: \Phi \rightarrow \mathbb{N}_{\geqslant 1}
$$

be the map that sends $\Phi_{k}(\mathrm{pt})$ to $\{k\}$. Together with $\theta$, these maps form the top square of the diagram.

The three commuting squares in the second row are almost immediate from the properties of $P$ : the right-most follows, by induction over the degree, from the exponential property. The unlabeled horizontal maps out of the tensor products on the very right all are obtained as the (internal) product of projection maps of the form

$$
S\left(\coprod_{k} Y_{k}\right) \longrightarrow S\left(Y_{j}\right),
$$

mapping $\left(y_{i}\right)$ to the list of entries from $Y_{j}$.

Next, we have the maps $\operatorname{diag}_{\Lambda \mathrm{X}}$ defined as

$$
\begin{aligned}
\operatorname{diag}_{\Lambda \mathrm{x}}: S \mathbb{N}_{\geqslant 1} \times \Lambda X & \longrightarrow S\left(\mathbb{N}_{\geqslant 1} \times \Lambda X\right) & & \\
\left(\left(k_{1}, \ldots, k_{n}\right), g\right) & \longmapsto\left(\left(k_{1}, g\right), \ldots,\left(k_{n}, g\right)\right) & & \text { on objects } \\
\left(\sigma_{n}, h\right) & \left.\longmapsto\left(\sigma_{n} ; h, \ldots, h\right)\right) & & \text { on morphisms }
\end{aligned}
$$

and similarly, with $\Phi$ in the role of $\mathbb{N}_{\geqslant 1}$. 
Finally, we write $\Pi$ for the set of isomorphism classes of $\Lambda S(\mathrm{pt})$ or, equivalently, $S \Phi$ or $S \mathbb{N}_{\geqslant 1}$. Elements of $\Pi$ may be thought of as sequences $\left(n_{k}\right)_{k \geqslant 1}$ with all but finitely many entries equal to zero, and we have the degree map

$$
\begin{aligned}
s: \Pi & \longrightarrow \mathbb{N}_{0} \\
\left(n_{k}\right)_{k} & \longmapsto \sum_{k} k n_{k},
\end{aligned}
$$

and the canonical quotient map

$$
e: S \mathbb{N}_{\geqslant 1} \longrightarrow \Pi
$$

fitting into the pentagon in the fourth row. The right square in the same row is obtained from the family of commuting squares

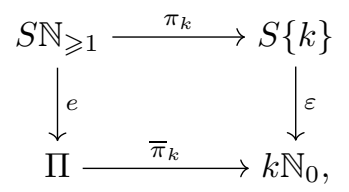

where $\bar{\pi}_{k}$ is the map of isomorphism classes induced by $\pi_{k}$. The fact that $e_{!} \circ \prod \pi_{k}^{*}$ equals $\otimes \varepsilon_{\text {! }}$ is not immediate. The argument boils down to the fact that, for a $G$-representation $V$ and an $H$-representation $W$, the invariant part $(V \otimes W)^{G \times H}$ equals $V^{G} \otimes W^{H}$.

\subsection{Adams operations and Hecke operators}

The total symmetric power in $K$-theory has the well-known generating function

$$
S_{t}(x)=\exp \left(\sum_{m \geqslant 1} \frac{\psi_{m}(x)}{m} t^{m}\right),
$$

where the $\psi_{m}$ are the Adams operations. In fact, (14) is often used as a definition of the Adams operators. As a consequence of Theorem 3.24, we obtain the following generating function for $S_{t}^{\text {Tate }}$ :

$$
S_{t}^{\text {Tate }}(F)=\exp \left(\sum_{m \geqslant 1} T_{m}(F) t^{m}\right),
$$

where the Hecke operators $T_{m}$ are defined as

$$
T_{m}(F):=\frac{1}{a} \sum_{a d=m} \psi_{a}\left(\beta_{d}(F)\right)
$$

Example 3.26. It is well known that the Adams operations on the coefficient rings $K_{G}(\mathrm{pt})=R(G)$ have the following effect on characters:

$$
\psi_{m}(\chi)(g)=\chi\left(g^{m}\right) .
$$

Let $F$ be an element of the coefficient ring

$$
K_{G}^{\text {Tate }}(\mathrm{pt}) \cong \bigoplus_{[g]} R\left(C_{g}\right) \llbracket q^{\frac{1}{|g|}} \rrbracket .
$$


As in Section 2.1.3, we view $F$ as the $q$-expansion of a function $F(g, h ; \tau)$, with $q^{\frac{1}{|g|}}=e^{2 \pi i \tau /|g|}$. Then

$$
\beta_{d}(F)(g, h ; \tau)=\frac{1}{d} \sum_{0 \leqslant b<d} F\left(g^{d}, g^{-b} h ; \frac{\tau+b}{d}\right),
$$

and hence

$$
T_{m}(F)(g, h ; \tau)=\frac{1}{m} \sum_{a d=m} \sum_{0 \leqslant b<d} F\left(g^{d}, g^{-b} h^{a} ; \frac{\tau+b}{d}\right) .
$$

These are the equivariant Hecke operators that play an important role in Moonshine; see $[\mathbf{1 2}, \mathbf{1 7}, \mathbf{2 7}]$ and $[\mathbf{2 9}]$.

The equivariant Hecke operators can be defined by an equivariant Hecke correspondence, and this is the sense in which our power operations on $K_{\text {Tate }}$ are 'elliptic'. A natural question is whether our notion of elliptic can be strengthened to resemble the definition of $H_{\infty}$ elliptic spectrum in [5, Def. 16.4]. In other words, do our power operations define descent data for level structures on the Tate curve as in [5], and do our power operations specialize to the ones Ando defines in $[\mathbf{2}, 6.3]$ ?

\subsection{Exterior powers and replicability}

The total exterior power $\Lambda_{t}^{\text {Tate }}$ is defined by the equality

$$
\Lambda_{t}^{\text {Tate }}(F)=\left(S_{-t}^{\text {Tate }}(F)\right)^{-1}
$$

so

$$
\Lambda_{-t}^{\text {Tate }}(F)=\exp \left(-\sum_{m \geqslant 1} T_{m}(F) t^{m}\right) .
$$

Let

$$
F(q) \in R(G)((q))
$$

be of the form

$$
F(q)=q^{-1}+a_{1} q+a_{2} q^{2}+\cdots .
$$

From the Moonshine literature, such $F$ are known as McKay-Thompson series. We recall $^{4}$ that the Faber polynomials $\Phi_{m, F}$ of $F$ are defined by

$$
-\sum_{m=1}^{\infty} \Phi_{m, F}(w) t^{m}=\log (t(F(t)-w)) .
$$

Hence $\Phi_{m, F}$ is a polynomial in $w$ of degree $m$, which depends on the first $m$ coefficients of $F$ and is uniquely characterized by the fact that it is of the form

$$
\Phi_{m, F}(F(q))=q^{-m}+b_{1} q+b_{2} q^{2}+\cdots .
$$

\footnotetext{
${ }^{4}$ Compare, e.g., $[35,(2.1)]$ with $b=1, t=1 / z$ and $F(q)=g(z)$.
} 
Definition 3.27. Let $F$ be a McKay-Thompson series. We write $F^{(a)}$ for the $a^{\text {th }}$ Adams operations applied to $F$. We call $F$ replicable, if for every natural number $m$, we have

$$
\begin{aligned}
\Phi_{m, F}(F(q)) & =\sum_{a d=m} \sum_{0 \leqslant b<d} F^{(a)}\left(\frac{a \tau+b}{d}\right) \\
& =m \cdot T_{m}(F)(q) .
\end{aligned}
$$

Here $q=e^{2 \pi i \tau}$.

This appears to be the right notion of replicability of McKay-Thompson series; it is the one that turns up in [11]. It follows that a McKay-Thompson series $F$ is replicable if and only if it satisfies

$$
F(t)-F(q)=t^{-1} \cdot \Lambda_{-t}^{\text {Tate }}(F(q))
$$

\section{References}

[1] M. Ando, Isogenies of formal group laws and power operations in the cohomology theories $E_{n}$, Duke Math. J. 79 (1995), no. 2, 423-485.

[2] M. Ando, Power operations in elliptic cohomology and representations of loop groups, Trans. Amer. Math. Soc. 352 (2000), no. 12, 5619-5666.

[3] M. Ando, The sigma orientation for analytic circle-equivariant elliptic cohomology, Geom. Topol. 7 (2003), 91-153 (electronic).

[4] M. Ando, M.J. Hopkins and N.P. Strickland, Elliptic spectra, the Witten genus and the theorem of the cube, Invent. Math. 146 (2001), no. 3, 595-687.

[5] M. Ando, M.J. Hopkins and N.P. Strickland, The sigma orientation is an $H_{\infty}$ map, Amer. J. Math. 126 (2004), no. 2, 247-334.

[6] M.F. Atiyah, Power operations in K-theory, Quart. J. Math. Oxford Ser. (2) 17 (1966), no. 1, 165-193.

[7] A. Baker, Hecke algebras acting on elliptic cohomology, in Homotopy theory via algebraic geometry and group representations (Evanston, IL, 1997), Contemp. Math. 220, 17-26, Amer. Math. Soc., Providence, RI, 1998.

[8] A. Baker, Hecke operators as operations in elliptic cohomology, J. Pure Appl. Algebra 63 (1990), no. 1, 1-11.

[9] A. Baker, Hecke operations and the Adams $E_{2}$-term based on elliptic cohomology, Canad. Math. Bull. 42 (1999), no. 2, 129-138.

[10] V. Baranovsky and V. Ginzburg, Conjugacy classes in loop groups and G-bundles on elliptic curves, Internat. Math. Res. Notices 15 (1996), 733751.

[11] R.E. Borcherds, Monstrous moonshine and monstrous Lie superalgebras, Invent. Math. 109 (1992), no. 2, 405-444.

[12] S. Carnahan, Generalized moonshine I: genus-zero functions, Algebra Number Theory 4 (2010), no. 6, 649-679. 
[13] P. Deligne and M. Rapoport, Les schémas de modules de courbes elliptiques, in Modular functions of one variable, II (Proc. Internat. Summer School, Univ. Antwerp, Antwerp, 1972), 143-316, Lecture Notes in Math. 349, SpringerVerlag, New York, 1973.

[14] J.A. Devoto, Equivariant elliptic homology and finite groups, Michigan Math. J. 43 (1996), no. 1, 3-32.

[15] R. Dijkgraaf, G. Moore, E. Verlinde and H. Verlinde, Elliptic genera of symmetric products and second quantized strings, Comm. Math. Phys. 185 (1997), no. 1, 197-209.

[16] N. Ganter, Orbifold genera, product formulas and power operations, Adv. in Math. 205 (2006), no. 1, 84-133.

[17] N. Ganter, Hecke operators in equivariant elliptic cohomology and generalized Moonshine, in Groups and symmetries, CRM Proc. Lecture Notes 47, 173-209, Amer. Math. Soc., Providence, RI, 2009.

[18] N. Ganter, Global Mackey functors with operations and $n$-special lambda rings, arXiv:1301.4616, 2013.

[19] V. Ginzburg, M. Kapranov, and E. Vasserot, Elliptic algebras and equivariant elliptic cohomology, arXiv:q-alg/9505012, 1995.

[20] J.P.C. Greenlees, Rational $S^{1}$-equivariant elliptic cohomology, Topology 44 (2005), no. 6, 1213-1279.

[21] F. Hirzebruch, T. Berger and R. Jung, Manifolds and modular forms, With appendices by N.P. Skoruppa and by P. Baum, Aspects of Mathematics E20, Friedr. Vieweg \& Sohn, Braunschweig, 1992.

[22] M.J. Hopkins, N.J. Kuhn and D.C. Ravenel, Generalized group characters and complex oriented cohomology theories, J. Amer. Math. Soc. 13 (2000), no. 3, 553-594 (electronic).

[23] E. Lupercio and B. Uribe, Loop groupoids, gerbes, and twisted sectors on orbifolds, in Orbifolds in mathematics and physics (Madison, WI, 2001), Contemp. Math. 310, 163-184, Amer. Math. Soc., Providence, RI, 2002.

[24] E. Lupercio, B. Uribe and M.A. Xicoténcatl, The loop orbifold of the symmetric product, J. Pure Appl. Algebra 211 (2007), no. 2, 293-306.

[25] M. Mahowald and M. Hopkins, The structure of 24 dimensional manifolds having normal bundles which lift to $B \mathrm{O}[8]$, in Recent progress in homotopy theory (Baltimore, MD, 2000), Contemp. Math. 293, 89-110, Amer. Math. Soc., Providence, RI, 2002.

[26] G. Mason, Orbifold conformal field theory and cohomology of the Monster, unpublished online notes.

[27] J. McKay, The essentials of Monstrous Moonshine, in Groups and combinatorics - in memory of Michio Suzuki, Adv. Stud. Pure Math. 32, 347-353, Math. Soc. Japan, Tokyo, 2001.

[28] I. Moerdijk, Orbifolds as groupoids: an introduction, in Orbifolds in mathematics and physics (Madison, WI, 2001), Contemp. Math. 310, 205-222, Amer. Math. Soc., Providence, RI, 2002. 
[29] J. Morava, Moonshine elements in elliptic cohomology, in Groups and symmetries, CRM Proc. Lecture Notes 47, 247-257, Amer. Math. Soc., Providence, RI, 2009.

[30] S.P. Norton, Appendix to 'Finite groups and modular functions' by Geoffrey Mason, in The Arcata Conference on Representations of Finite Groups (Arcata, Calif., 1986), Proc. Sympos. Pure Math. 47, 181-210, Amer. Math. Soc., Providence, RI, 1987.

[31] A. Pressley and G. Segal, Loop groups, Oxford Mathematical Monographs, Oxford Science Publications, The Clarendon Press, Oxford University Press, New York, 1986.

[32] C. Rezk, Lectures on the Tate curve, unpublished notes.

[33] N.J. Stapleton, Transchromatic generalized character maps, arXiv:1110.3346, 2012.

[34] H. Tamanoi, Infinite product decomposition of orbifold mapping spaces, Algebr. Geom. Topol. 9 (2009), no. 1, 569-592.

[35] L.P. Teo, Analytic functions and integrable hierarchies - characterization of tau functions, Lett. Math. Phys. 64 (2003), no.1, 75-92.

Nora Ganter nganter@unimelb.edu.au

Department of Mathematics and Statistics, The University of Melbourne, Parkville, Victoria, 3010, Australia 